\title{
USOS SOCIOECONÓMICOS DE LAS TIC RELACIONADOS CON EL EMPLEO EN EUROPA
}

\section{Begoña Ballesteros-Carrasco}

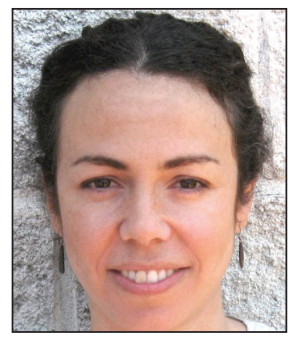

Begoña Ballesteros-Carrasco es doctora en Comunicación, Cambio Social y Desarrollo por la Universidad Complutense de Madrid (UCM). Miembro del grupo de investigación consolidado de la UCM Identidades Sociales y Comunicación. Colabora como docente en la asociación Entrenadores de Pensamiento y forma parte de la junta directiva de la sección española de la Unión Latina de Economía Política de la Información, la Comunicación y la Cultura (Ulepicc). http://orcid.org/0000-0002-6434-5847

Grupo de investigación Identidades sociales y comunicación Facultad de Ciencias de la Información, Universidad Complutense de Madrid Avenida Complutense s/n. 28040 Madrid, España begoballesteros@ccinf.ucm.es

\section{Resumen}

Las tecnologías de la información y de la comunicación (TIC) suelen asociarse a mejoras socioeconómicas y se han vinculado con la creación de empleo o con aumentos en la calidad y retribución del trabajo. Sin embargo a nivel macroeconómico llama la atención que mientras el proceso de digitalización aumenta, el empleo y los salarios están deteriorándose en términos generales. Se exploran, con datos referidos a la UE-15, algunos usos de estas tecnologías en relación con el paro y los salarios, en un contexto económico marcado por problemas crecientes de rentabilidad y por el auge de los mercados financieros.

\section{Palabras clave}

TIC, Tecnologías de la información, Trabajo, Crisis económica, Capitalismo, Informatización, Mercados, Profesiones.

\section{Title: Socioeconomic uses of ICT related to employment in Europe}

\begin{abstract}
Information and communication technologies (ICT) are often related to economic and social improvements. They have been associated with job creation as well as increases in the quality of work and remuneration. However, at a macroeconomic level, it is noteworthy that while the digitization process increases, employment and wages are generally deteriorating. Referencing EU-15 data, this article explores some uses of these technologies in relation to unemployment and wages, within an economic context marked by increasing problems of profitability and by the rise of financial markets.
\end{abstract}

\section{Keywords}

ICT, Information technologies, Labour, Professions, Economic crisis, Capitalism, Computerization, Markets.

Ballesteros-Carrasco, Begoña (2013). “Usos socioeconómicos de las TIC relacionados con el empleo en Europa”. El profesional de la información, julio-agosto, v. 22, n. 4, pp. 304-308.

http://dx.doi.org/10.3145/epi.2013.jul.05

\section{Informatización y trabajo ${ }^{1}$}

Las tecnologías de la información y la comunicación (TIC) suelen asociarse habitualmente con avances económicos y sociales. Algunas investigaciones, como la coordinada por Torrent-Sellens (2008), han establecido vínculos entre inversión en TIC y creación de empleo, y también entre digitalización de empresas y mejoras salariales en algunos perfiles profesionales.

Sin embargo existen datos macroeconómicos que invitan a revisar esta relación positiva entre equipos informáticos y condiciones de trabajo de la población asalariada en la Europa de los quince (UE-15). Como punto de partida se puede identificar la siguiente paradoja:

- Por un lado las tecnologías digitales se han incorporado al funcionamiento de las empresas y al quehacer cotidiano de sus empleados.

Según la Encuesta europea sobre las condiciones de trabajo (EECT) que realiza Eurofound, la mayor parte del empleo se ha informatizado desde los años 90. En 2010 un $83,2 \%$ de los profesionales muy cualificados y casi un $65 \%$ de los profesionales de nivel medio usaban habitualmen- 
te estos dispositivos para trabajar. Incluso en los trabajadores manuales, con porcentajes más bajos, la cifra se ha incrementado en más de un 6\% desde 1995.

-Por otro lado junto a esta progresiva digitalización, el empleo y las rentas de los trabajadores han sufrido una evolución claramente decreciente desde los años 80 hasta la actualidad.

Según la base de datos macroeconómica de la Comisión Europea (Ameco), el paro ha pasado de un $2 \%$ de la población activa en 1970 a cerca de un 10\% en 2010, convirtiéndose en un problema estructural. El deterioro salarial se constata en la participación decreciente de los sueldos en el producto interior bruto (PIB). En 1975 las rentas de los trabajadores de toda la UE-15 llegaron a suponer un $67,3 \%$ del PIB, descendiendo paulatinamente hasta un $58,4 \%$ en 2010 . Esta tendencia a la baja está referida a la tasa salarial general y por tanto es compatible con que se hayan producido mejoras retributivas en algunas categorías de fuerza de trabajo más demandadas y valoradas que el resto. De hecho si se atiende a los sectores más tecnificados, los datos se suavizan, pero la paradoja no desaparece. El paro en perfiles que hacen un uso más intensivo en TIC es siempre menor, pero tiene una evolución similar al del resto de la economía, incluso con aumentos más rápidos en algunas coyunturas como tras la crisis bursátil de las empresas "punto com" a partir de 2001.

\section{Hay que preguntarse por el papel de las TIC en el actual deterioro del empleo y de los salarios}

En este escenario de avance de la informatización y deterioro del empleo y los salarios, es necesario proponer nuevas interpretaciones sobre el papel de las TIC en la dinámica macroeconómica europea. La revolución digital no es la causa directa ni principal de la evolución laboral descrita. En términos históricos la aparición de las TIC ha supuesto una mejora objetiva para el trabajo y su productividad: con ellas se han podido modernizar las plantas de producción, reducir los perfiles laborales obsoletos y eliminar algunas tareas repetitivas o peligrosas asociadas al modelo de fábrica fordista ${ }^{2}$.

Sin embargo estas tecnologías no son ajenas a las dinámicas macroeconómicas referidas. Por tanto cabe preguntarse cómo son utilizadas en las empresas a tenor de la crisis de la economía europea desde los años 70. Algunos economistas como Vidal-Villa y Martínez-Peinado (2000) o Sanabria (2009) han mostrado que es entonces cuando el capitalismo comienza a manifestar dificultades estructurales para obtener rentabilidad. Estas dificultades no parecen haber revertido hasta la fecha; han existido coyunturas favorables, pero ninguna ha dado paso a una nueva etapa de crecimiento sostenido.

En este trabajo se proponen dos explicaciones apoyadas en datos estadísticos sobre los usos socioeconómicos de las TIC: 1) relacionados con el crecimiento del paro y con la necesidad de las empresas de mejorar sus beneficios a corto plazo; y 2) relacionados con el aumento del trabajo no pagado al empleado y con la necesidad de las empresas por obtener más plusvalía de los procesos productivos.

\section{Beneficios, TIC y desempleo}

Para aproximarse a los usos de las TIC en el empleo hay que tener presentes algunas claves del escenario económico de los últimos treinta años y particularmente el predominio ascendente de los mercados financieros. El impulso a estos mercados, con su paulatina desregulación y apertura, fue una de las medidas adoptadas tras la crisis de los 70 para frenar los problemas de rentabilidad del sistema. Actualmente el crédito se ha extendido como nunca, las principales bolsas mueven valores muy superiores a la riqueza de algunos países y el volumen de las transacciones financieras ha crecido más rápido que las actividades productivas o comerciales. Según cifras de Álvarez y Luengo (2010), la tasa de financiarización ${ }^{3}$ en la UE-15 superaba el $22 \%$ en 2009 cuando en 1975 estaba por debajo del 10\%.

El origen especulativo de la actual crisis económica, que estalla en 2007, puso de manifiesto la relevancia adquirida por este sector. Como constatan Álvarez y Luengo (2010), el capital financiero está logrando imprimir su lógica operativa al resto de la economía y particularmente al funcionamiento cotidiano de las empresas no financieras. Un efecto que conviene resaltar por su importancia es la prioridad que las grandes empresas dan a sus beneficios a corto plazo en las últimas décadas. La presión de los inversores obliga a aumentar el valor accionarial de una compañía por encima de cualquier otro objetivo. Para ello se requiere obtener beneficios tan inmediatos como sea posible, incluso descartando estrategias productivas o comerciales que renten ganancias a más largo plazo. La tasa de acumulación, que es un indicador de las inversiones productivas, ha descendido en más de tres puntos porcentuales entre 1973 y 2009 (Álvarez; Luengo, 2010). Y los beneficios empresariales se han incrementado en promedio un 38\% entre 1999 y 2009 (Navarro, 2007). Si la tasa de beneficio empresarial estaba cercana al $60 \%$ en 1981, diez años después ya había superado el $80 \%$ y veinte años más tarde, en los primeros años 2000, rondaba el $100 \%$.

Este auge de la economía especulativa no sólo dificulta la creación de nuevo empleo (al desincentivar la actividad productiva), sino que parece premiar estrategias de reducción de costes laborales como manera de obtener beneficios cortoplacistas. Los inversores suelen respaldar las reestructuraciones empresariales que impliquen abandonar líneas de negocios poco rentables o reducir el tamaño de las empresas, aunque eso implique (o precisamente porque eso implica) un recorte en las contrataciones. De hecho las decisiones sobre recursos humanos que toman los directivos están condicionadas desde la esfera especulativa. En cuestión de horas la reacción de los accionistas puede disparar o hundir la cotización bursátil de una firma, avalando o sancionando cualquier decisión respecto de sus recursos humanos (Álvarez; Medialdea, 2010). Las bolsas suelen ser sensibles a este tipo de fluctuaciones. Como ya advirtió Forrester en 2000, sus valores suben cuando se anuncian despidos y caen ante 
repuntes en el empleo. En definitiva la prioridad por los beneficios inmediatos y unas legislaciones laborales cada vez menos proteccionistas, han convertido al empleo en un elemento cada vez más elástico, a merced de reducciones que puedan ayudar a mejorar la cuenta de resultados.

En este escenario las TIC son útiles para algunas empresas porque ayudan a flexibilizar la plantilla de personal en momentos críticos. Esto concuerda en primer lugar con la constatación empírica de que la inversión en bienes de equipo digitales es mayor en los países europeos con menores restricciones legales en materia de despidos y contratación (Pérez, 2004; Barrios; Burgelman, 2007). Una legislación proteccionista parece repercutir negativamente en las decisiones de las empresas para incorporar sistemas digitales.

Las tecnologías digitales automatizan el trabajo vivo y han hecho posible la renovación de tareas y ocupaciones obsoletas. Sin embargo en los últimos años se dispone de datos que reflejan un uso de las TIC orientado a prescindir de perfiles cualificados, con puestos técnicos o administrativos, que no se han quedado técnicamente obsoletos. La carga laboral de esos empleados se traspasa a otros individuos que permanecen en la compañía: hay menos gente contratada que tiene que trabajar más. Un fenómeno en el que los dispositivos informáticos están sirviendo para incrementar tanto la carga laboral individual como la versatilidad del empleado. Con estos usos, se contribuye a la reorganización del volumen total de trabajo entre un número menor de empleados. Esto sucede por ejemplo cuando una compañía mantiene o incluso eleva su volumen de facturación al tiempo que debe prescindir de una parte de su fuerza de trabajo para no comprometer el nivel de beneficios esperados.

Los indicadores de intensidad laboral han crecido paulatinamente en aquellos perfiles profesionales más informatizados

Una forma de elevar la carga laboral entre menos personas consiste en aumentar la intensidad de la prestación y la exigencia de los objetivos encomendados a cada trabajador. De ambos fenómenos hay datos vinculados a las TIC. Ahora es cada vez más frecuente que el rendimiento laboral se mida por el resultado y la calidad de la tarea, de manera que la cantidad de trabajo real empleado se obvie en el contrato. Entre 1995 y 2010, el crecimiento más espectacular de estos esquemas de trabajo en base a metas es de un $20 \%$ y se da precisamente entre las ocupaciones que más usan TIC: en 2010 alcanzaba ya al $48 \%$ de estos profesionales altamente cualificados, según la EECT.

En cuanto a la intensidad laboral, trabajar con ordenadores portátiles o con teléfonos conectados a la red hace posible minimizar tiempos muertos y acelerar el ritmo de las tareas tanto como sea preciso. Los indicadores de intensidad laboral han crecido paulatinamente en aquellos perfiles profesionales más informatizados. Cada vez más personas trabajan más deprisa, con plazos más ajustados y sin tiempo suficiente para hacer bien sus tareas (Eurofound, 2009). De acuerdo con la $E E C T$, el $56,7 \%$ de las ocupaciones que hacen un uso más intensivo de las TIC afirmaba trabajar a un ritmo muy rápido en 2010,6\% más que en 1995 . En los técnicos y administrativos de media y baja cualificación (los segundos que más recurren a las TIC), los porcentajes son similares con un incremento de $8 \%$ en esos dos años. Respecto de los plazos de entrega la evolución es similar: aquellos que tienen su trabajo más informatizado son quienes más trabajan, con márgenes de tiempo exigentes en al menos una cuarta parte de sus actividades. En 2010 eran ya un 68,6\% del total de los profesionales muy cualificados, 7 puntos más que en 1995.

\section{Las TIC están sirviendo para ayudar a au- mentar el tiempo de trabajo no pagado}

Las TIC también pueden usarse para aumentar la versatilidad de la plantilla cuando hay que reestructurarla y reducirla. La informática ha facilitado que un solo empleado pueda resolver labores administrativas, de gestión, ejecutivas o intelectuales, de manera que se puede encomendar a menos personas tareas de diversa índole antes repartidas entre más perfiles especializados. Entre 2005 y 2010, el número de personas que tiene que hacer rotación de tareas y habilidades en su puesto de trabajo ha aumentado entre los perfiles ocupacionales que más usan las TIC, como muestra la EECT: casi $3 \%$ en técnicos y administrativos de cualificación media, y cerca de $1,5 \%$ en profesionales altamente cualificados. Como contraste, entre los trabajadores que menos recurren a las TIC, el grado de rotación es el menor de todos y apenas ha aumentado entre esos dos años.

\section{Plusvalía, TIC y tiempo de trabajo}

El aumento de los beneficios financieros y de los movimientos especulativos no están revirtiendo los problemas estructurales de rentabilidad de la economía, sino más bien agudizándolos. La reducción de costes laborales y los ingresos financieros permiten mejorar la cuenta de resultados a corto plazo, pero no crean riqueza o valor real. Como se sabe, en condiciones capitalistas el valor sólo se genera en los procesos productivos. En concreto se origina en aquella parte del trabajo efectuado que no se remunera al asalariado. De este sobretrabajo o trabajo no pagado, el empresario extrae la plusvalía que necesita para que su negocio tenga rentabilidad real (no especulativa). Se entiende que la obtención de esa rentabilidad se dificulta cuando los procesos productivos y la nueva contratación se estancan o decrecen, como así está sucediendo.

Las TIC también parecen implicadas en esta necesidad cada vez más acuciante por extraer plusvalía de los procesos productivos porque pueden repercutir en el aumento del tiempo de trabajo no pagado. Los datos reflejan dos tipos de usos de las TIC orientados a ese fin:

- Elevar la productividad del empleo sin un acortamiento de la jornada laboral o una elevación del salario. De aquí se obtiene lo que la economía política llama "plusvalía relativa". 
- Ayudar a prolongar la jornada laboral. De aquí se extrae la "plusvalía absoluta".

Los datos sobre productividad en Europa siguen siendo bajos, pero ni el sueldo ni las horas de trabajo han mejorado en proporción a los incrementos de la producción media por empleado desde la digitalización de la economía (plusvalía relativa). Por ejemplo en España, donde la productividad creció un $2,1 \%$ entre 2001 y 2005 , los salarios cayeron hasta un -0’9\% medio anual (Sanabria, 2009). Este fenómeno, propiciado por otros factores políticos y económicos, ha confirmado la ruptura de la norma que durante el fordismo vinculaba la remuneración del trabajo con la evolución de la productividad. Los datos sobre plusvalía absoluta reflejan una implicación de las TIC en la prolongación de la jornada laboral tanto dentro como fuera de la oficina. En el epígrafe anterior ya se ha mencionado que el trabajo se acrecienta cuanto más intenso es. Pero además los equipos digitales pueden ayudar a alargarlo en el tiempo. Esto ocurre sobre todo cuando las TIC se emplean para trabajar en espacios y horarios no laborales sin reconocerse en el contrato. Suele suceder cuando se está sometido a objetivos exigentes y entre empleados altamente cualificados e informatizados (incluso con cargos directivos). Un estudio de Vaguer y VanBastelaer (2004) para la UE-15 ha cuantificado que las ocupaciones que más usan TIC son aquellas que presentan un número más elevado de horas extra no pagadas. La media está en 7 horas extras semanales, mientras que las que se remuneran son levemente superiores a 2 horas. Según desciende la formación y el grado de digitalización del trabajo, los promedios de horas extra no pagadas disminuyen y las retribuidas aumentan. En los trabajos manuales, cualificados o no, el promedio de horas extra no pagadas nunca llega a las tres. En esta línea, un $22 \%$ de los directivos europeos reconocía en 2004 que la flexibilidad espaciotemporal de sus empleados, facilitada con las TIC les permitía reducir el dinero destinado a pagar horas extra (Riedman, 2006) ${ }^{4}$.

El uso de las TIC puede provocar que la vida productiva termine articulando todos los espacios y tiempos, tanto sociales como personales, en los que discurre la vida del individuo (Martín-Serrano, 1992)

A veces este sobretrabajo es obligatorio. Por ejemplo, cuando se convocan reuniones virtuales fuera de los horarios estipulados por contrato. Otras veces es opcional pero conveniente, sobre todo si el asalariado desea promocionarse o-en casos extremos- evitar ser despedido en una reestructuración de personal como las mencionadas. En todo caso parece un fenómeno cada vez más extendido. Una parte creciente del teletrabajo digital no se está pagando ni reconociendo contractualmente, como algunas investigaciones nacionales están corroborando (Eurofound, 2010). Por ejemplo en Holanda los individuos que trabajan en casa mediante TIC lo hacen sobre todo en fines de semana y noches, fuera de las horas de trabajo (Graaff; Rietveld, 2003).
Este uso de las TIC para ayudar a prolongar el trabajo puede provocar que la vida productiva termine articulando todos los espacios y tiempos, tanto sociales como personales, en los que discurre la vida del individuo (Martín-Serrano, 1992). Un rasgo característico de este uso de las tecnologías informacionales es el llamado "problema de la no desconexión". En la EECT de 2005 se refleja que las personas que teletrabajan desde sus hogares están más condicionadas a permanecer conectadas al trabajo fuera de sus horas de oficina y a recibir llamadas o correos electrónicos. Las instituciones europeas reconocen la gravedad de esta incapacidad para desconectar pero se suele apelar a la autodisciplina como forma de control (Plantenga; Remery, 2010). Con los sistemas informáticos se podrían articular mecanismos de conexión y desconexión obligatorios para reconocer o prevenir el sobretrabajo. Ese sería un ejemplo de uso de las TIC que comportaría efectos beneficiosos para el trabajador. Sin embargo podría dificultar el objetivo último de incrementar la rentabilidad empresarial por lo que no es fácil que se generalice.

Las tecnologías digitales están contribuyendo a remontar los márgenes globales de ganancia que necesita el sistema económico para seguir funcionando

\section{Conclusión}

En los últimos años las instituciones políticas y económicas europeas han promovido la informatización de las empresas para avanzar en la llamada economía del conocimiento. Sin embargo no exigen que ese desarrollo vaya asociado a la creación de empleo o al crecimiento de las inversiones productivas. Más bien al contrario, cuando se desregula el trabajo y no se pone cota al poder de las finanzas, la digitalización corre el riesgo de utilizarse para mejorar la competitividad mediante el empeoramiento del empleo y de los salarios. Las TIC se están convirtiendo en un componente más de un modelo económico donde por un lado los beneficios empresariales están creciendo a costa entre otras cosas de no crear empleo, y por otro lado la eficiencia productiva se está logrando a costa, entre otros factores, de peores condiciones laborales.

Este deterioro histórico del trabajo no debe entenderse como fruto inherente al desarrollo tecnológico digital, sino como el resultado de una sociedad capitalista en crisis, que requiere supeditar sus avances científico-técnicos al incremento del beneficio económico. En términos globales y con las excepciones de rigor, los datos apuntan a que las formas de usar y aplicar las tecnologías digitales están contribuyendo a remontar los márgenes globales de ganancia que necesita el sistema económico para seguir funcionando.

Dicha conclusión requerirá de mayor investigación pero permite entender por el momento por qué las TIC, a pesar de su potencial para estimular la contratación, generar más riqueza y facilitar que se trabaje menos y mejor, se están viendo implicadas en las mayores cifras de paro en Europa 
desde la 2ạ Guerra mundial y en una precarización generalizada de las condiciones de vida y trabajo de la mayoría de la población.

\section{Notas}

1. Este texto se ha elaborado a partir de la tesis doctoral de la autora (Ballesteros-Carrasco, 2012).

2. Nota del editor. Fordismo se refiere al modo de producción en cadena que llevó a la práctica Henry Ford, fabricante de automóviles de EUA.

http://es.wikipedia.org/wiki/Fordismo

3. Nota del editor. Financiarización es un proceso económico que intenta obtener beneficio mediante canales financieros en vez de mediante la producción y comercialización de bienes y servicios no especulativos.

4. Este dato procede de una encuesta realizada en la UE-21 y no en la UE-15.

\section{Bibliografía}

Álvarez, Ignacio; Luengo, Fernando (2010). Financiarización, empleo y salario en la UE: el impacto de las nuevas estrategias empresariales. Madrid: Instituto Complutense de Estudios Internacionales.

http://eprints.ucm.es/10599

Álvarez, Ignacio; Medialdea, Bibiana (2010). "La influencia de la financiarización sobre el gobierno corporativo de la empresa: el papel de los inversores institucionales". Revista de economía mundial, n. 24, pp. 165-191.

http://rabida.uhu.es/dspace/bitstream/handle/10272/3579/ b15777911.pdf?sequence $=1$

Ballesteros-Carrasco, Begoña (2012). Las tecnologías de la información y de la comunicación en los cambios laborales: análisis sociohistórico de 1970 a 2010. Tesis doctoral. Universidad Complutense de Madrid.

http://eprints.ucm.es/16168/1/T33820.pdf

Barrios, Salvador; Burgelman, Jean-Claude (2007). Information and communication technologies, market rigidities and growth: Implications for EU policies. Luxemburgo: Oficina de Publicaciones de la Unión Europea. ISBN: 9789279074585 http://ftp.jrc.es/EURdoc/eur23027en.pdf http://dx.doi.org/10.2791/46759

Comisión Europea. Anual macro-economic database (Ameco). http://ec.europa.eu/economy_finance/db_indicators/ ameco/index_en.htm

Eurofound (2008). Condiciones de vida y de trabajo en Europa. http://www.eurofound.europa.eu/pubdocs/2008/90/es/1/ EF0890ES.pdf

Eurofound (2009). Comparative analysis of working time in the European Union. http://www.eurofound.europa.eu/docs/ewco/tn0803046s/ tn0803046s.pdf

Eurofound (2010). Encuesta europea sobre las condiciones de trabajo.

http://www.eurofound.europa.eu/surveys/smt/ewcs/results.htm

Forrester, Viviane (2000). Una extraña dictadura. Buenos Aires: Fondo de Cultura Económica. ISBN: 9505573588

Graaff, Thomas; Rietveld, Piet (2003). "ICT and substitution between out-of-home and at-home work". Tinbergen Institute discussion paper. Amsterdam: Tinbergen. http://www.tinbergen.nl/discussionpapers/03061.pdf

Martín-Serrano, Manuel (1992). “Los cambios acontecidos en las funciones de la comunicación y en el valor de la información". Revista española de investigaciones sociológicas, n. 57, pp. 13-20.

http://eprints.ucm.es/13240

Navarro, Vicenç (2007). “¿Cómo está evolucionando la situación social de la Unión Europea?”. Colección Mediterráneo económico, n. 12, pp. 417-437.

http://www.publicacionescajamar.es/pdf/publicacionesperiodicas/mediterraneo-economico/12/12-209.pdf

Pérez, Jorge (coord.) (2004). Productividad, crecimiento económico y TIC.

http://www.usc.es/atpemes/IMG/pdf/04_03_30productividad crecimiento_TIC.pdf

Plantenga, Janneke; Remery, Chantal (2010). Flexible working time arrangements and gender equality. A comparative review of 30 European countries. Luxemburgo: Oficina de Publicaciones de la Unión Europea. ISBN: 9789279155451 http://goo.gl/rEjDd

http://dx.doi.org/10.2767/29844

Riedman, Arnold (2006). Working time and work-life balance in European companies. Establishment survey on working time 2004-2005. Luxemburgo: Oficina de Publicaciones de la Unión Europea. ISBN: 9289709294

http://www.eurofound.europa.eu/pubdocs/2006/27/en/1/ ef0627en.pdf

Sanabria, Antonio (2009). "La naturaleza salarial del ajuste". En: Álvarez, Ignacio et al. (2009). Ajuste y salario. Las consecuencias del neoliberalismo en América Latina. Madrid: Fondo de Cultura Económica, pp. 27-51. ISBN: 9788437506241

Torrent-Sellens, Joan (coord.) (2008). La empresa red: tecnologías de la información y la comunicación, productividad y competitividad. Madrid: Ariel. ISBN: 9788434442771

Vaguer, Christine; Van-Bastelaer, Aloïs (2004). Working overtime. http://www.eds-destatis.de/en/downloads/sif/nk_04_11.pdf

Vidal, José-María; Martínez, Javier (2000). Economía mundial. Madrid: McGraw Hill. ISBN: 8448116844 